\section{Hesperidin Modulates Troponin-1 Serum Level and Decrease Heart Tissue Injury of Irradiated Rats}

\author{
Somayeh Sajadi ${ }^{1 \oplus}$, Gholamhassan Haddadi ${ }^{2,3 * \odot}{ }^{\circ}$, Fatemeh \\ Kadivar $^{1,4}$, Reza Fardid ${ }^{2,3}$
}

\begin{abstract}
Background: The heart is the major dose-limiting organ for radiotherapy of malignant tumor in the mediastanal region.

Objective: This study aims to investigate the radio protective effects of Hesperidin (HES) as a natural flavonoid after localized irradiation of the rat's mediastinum region.

Material and Methods: In this experimental study, we divided sixty male rats into 4 groups ( $\mathrm{n}=15)$. First group: Sham which received PBS; second group: Hesperidin only ( $100 \mathrm{mg} / \mathrm{kg} /$ day orally) for one week; third group: Radiation that received single dose of 20 Gy gamma radiation using Co-60 unit and the forth group: Radiation+HES that underwent the same dose of radiation and received HES for 7 days prior irradiation. Each group was divided in two branches. Early sampling from subgroup one was done 4-6 hours after irradiation to determine troponin-1 level changes. Rats of second subgroups were killed 56 days after irradiation for histopathological evidence.

Results: In radiation group, troponin -1 serum level had a significant increase in comparison with sham group $(\mathrm{P}<0.05)$. Histopathological evaluation of second subgroup showed there was a significant difference between sham and radiation group in some parameters. Inflammation $(\mathrm{p}=0.008)$, pericardial effusion $(\mathrm{P}=0.001)$, and vascular plaque $(\mathrm{P}=0.001)$ had an increase in the irradiation group. Oral administration of hesperidin significantly decreased all the above factors when was compared with irradiation group $(\mathrm{P}>0.016)$.
\end{abstract}

Conclusion: Oral administration of Hesperidine for seven days prior radiotherapy may decrease troponin-1 and cardiac injury due to radiation.

Citation: Sajadi S, Haddadi Gh, Kadivar F, Fardid R. Hesperidin Modulates Troponin-1 Serum Level and Decrease Heart Tissue Injury of Irradiated Rats. J Biomed Phys Eng. 2021;11(3):377-388. doi: 10.31661/jbpe.v0i0.969.

\section{Keywords}

Radiation; Heart Injury; Hesperidin

\section{Introduction}

arcinoma and cardiovascular diseases are the two major reasons for death in the world [1]. Radiotherapy is a powerful treatment to improve the survival rate of individuals who have suffered from cancer. In spite of the advantages, there are some side effects. When the cancerous site is irradiated, some healthy tissues are exposed to radiation; then the activity of the tissue is affected [2].
${ }^{1} \mathrm{MSc}$, Department of Radiology, Faculty of

Paramedicine, Shiraz

University of Medical Sci-

ences, Shiraz, Iran

${ }^{2} \mathrm{PhD}$, Department of

Radiology, Faculty of

Paramedicine, Shiraz

University of Medical Sci-

ences, Shiraz, Iran

${ }^{3} \mathrm{PhD}$, lonizing and

Non Ionizing Radiation

Protection Research

Center, Shiraz University

of Medical Sciences,

Shiraz, Iran

${ }^{4} \mathrm{MSc}$, lonizing and

Non lonizing Radiation

Protection Research

Center, Shiraz University

of Medical Sciences,

Shiraz, Iran

*Corresponding author:

Gholamhassan Haddadi

Department of Radiol-

ogy, Faculty of Paramedi-

cine, Shiraz University

of Medical Sciences,

Shiraz, Iran

E-mail: ghadadi@gmail.

com

Received: 25 June 2018

Accepted: 20 August 2018 
Radiation-induced heart damage is one of the life-threatening side effects of radiotherapy. By the mid-1960s, when a large amount of information for Hodgkin's lymphoma patients, who were treated with high doses of radiation, was obtained, the heart was known as an organ resistant to radiation. Based on many clinical studies and experiments were conducted, it has been indicated that the heart is one of the critical organs in radiotherapy. Heart disease after radiotherapy is one of the main long-term effects in survivors of Hodgkin's lymphoma, breast and lung cancers that can arise in the following statements: Pericardial, myocardial, Valvular and Coronary artery diseases, Conduction abnormalities, and cardiovascular death.

One of the mechanisms used to reduce normal tissue damage is the use of radioprotectors. If before or immediately after radiation therapy the Radioprotectors exist in the environment, the healthy tissues' response changes. The best known Radioprotectors are Sulfhydryl compounds such as cysteine and Cystamine. However, these compounds can cause serious side effects such as nausea and vomiting and have considerable toxicity in required doses for radiation protection.

The ability of some materials in reducing damage caused by ionizing radiation was first released in 1949 [3]. Research on radio-protectors with low toxicity has led to developing interest in natural products such as citrus extracts containing high levels of Flavonoids [4]. Flavonoids in fruits and vegetables are a family of polyphenol compounds. Hesperidin (HES), a glycoside flavone, belongs to the family of flavonoids, which are abundant in citrus fruits [5].

HES $\left(\mathrm{C}_{18} \mathrm{H}_{34} \mathrm{O}_{15}\right)$ is a cheap and abundant product in citrus cultivation and the main flavonoids exist in orange and lemon. In young immature oranges, fresh fruit constitutes more than $14 \%$ of HES [6].

Common standards for monitoring the heart during cancer treatment are based on the left ventricular ejection fraction showing cardiac damage only when it occurs and does not allow preventive measures. It has been proved that one of the ways to investigate the extent of damage to the heart is measuring cardiac biomarker levels. Cardiac biomarkers are substances released into the blood when the heart is damaged. Troponin I (TnI) is a specific cardiac biomarker that does not exist freely in the blood. In the heart attack, it is released from the heart, skeletal muscles and its rate rises in the serum. Since 2000, Europe Heart Association and American College of cardiologists have accepted cardiac troponin's role as a specific heart biomarker $[7,8]$.

Reports show that HES has many pharmacological effects, including anti-allergic, antioxidant, hypolipidimic, anti-inflammatory and anticarcinogen roles $[9,10]$. In general, citrus Bioflavonoids containing HES seem incredibly safe and without side effects even during pregnancy [11]. On the other hand, it is known that TnI is used as a biomarker to predict the heart damage [12]. However, there was no article about the radioprotective role of HES on heart and TnI serum levels; thus, we were prompted to do this research.

\section{Material and Methods}

\section{Chemicals}

In this experimental study, HES and phosphate buffered saline (PBS) tablet were purchased from Sigma Chemical Co. (St. Louis, MO, USA).

\section{Animals}

Healthy adult male Sprague-Dawley rats were purchased from Center of Comparative and Experimental Medicine, Shiraz University of Medical Sciences (SUMS), Shiraz, Iran. The rats, weighing 220-250 g, were housed based on the guidelines of "The Guide for The Care and Use of Laboratory Animals" prepared by SUMS in the university animal house. These principles include characteristics of animal's 
Hespiridin Modulates Heart Tissue Injury of Irradiated Rats

natural life in captivity situation using spacious cage, preparing appropriate ventilation and light, handling with care and giving water and standard pellet diet. All animals were kept under controlled conditions of humidity $(55 \pm 5 \%)$, temperature $\left(25 \pm 2{ }^{\circ} \mathrm{C}\right)$, and light (12 h of light and dark cycle). Four animals were housed together in polypropylene cages, which contain sterile husk bedding throughout the experiments. In the end, the ethics committee of SUMS approved this study.

\section{Irradiation of animals}

Before radiation exposure, the rats were anesthetized using ketamine 10\% (Alfasan, Woerden, Holland) and xylazine 2\% (Alfasan, Woerden, Holland) at a dose of $80 \mathrm{mg} / \mathrm{kg}$ and $5 \mathrm{mg} / \mathrm{kg}$, respectively, with an intraperitoneal injection. The rats were in the supine position and immobilized by taping the extremities on a well ventilated Plexiglas container. They were $\gamma$-irradiated with ${ }^{60} \mathrm{Co}$ unit at a source to surface distance of $55 \mathrm{~cm}$. A single dose of 20 Gy was delivered into the mediastinum at a dose in the department of RT, Namazi teaching Hospital, Shiraz, Iran. This dose was selected based on the data reported by Serin et al. The efficacy of melatonin as a radioprotector against radiotherapy induced acute lung damage, which was histopathologically evaluated in a rat model [13].

\section{Administration of HES}

HES was dissolved in PBS (pH 7.6) and administered orally using a balltipped needle for 7 consecutive days before exposure to irradiation and the drug was freshly prepared each day. To obtain optimum radiation protective effect of HES, the dose of $100 \mathrm{mg} / \mathrm{kg}$ was selected for present study according to the reports submitted by Hosseinimehr et al. and Pradeep et al. [14, 15]. They have shown that, as compared to the other doses of HES, this dose has better protective effect against radiation, which caused damage. To prepare this dose, $22 \mathrm{mg}$ of HES was dissolved in $2 \mathrm{ml}$ of
PBS.

\section{Experimental design}

The rats were irradiated with single fraction 20 Gy $\gamma$-radiation using a cobalt-60 unit, and the dose of HES was $100 \mathrm{mg} / \mathrm{kg} / \mathrm{d}$ orally for 7 days before irradiation. Each group was divided into two branches. Sampling of rats in branch A was done about 4-6 hours after irradiation. The samples were sent to laboratory for determination of TnI serum level changes as a cardiac biomarker.

The remaining animals (Branch B) were sacrificed 8 weeks after radiotherapy for histopathological evaluation.

\section{Sampling and tests}

In order to obtain blood from the animals, we placed them in a closed container containing Ether gas. Then, we entered the needle number 28 into the animal's heart for direct ventricular blood sampling and $2 \mathrm{ml}$ blood sample was taken. Samples of each rat were separately poured slowly (to prevent the hemolysis) within a test tube of gel. Then, these tubes were placed inside the centrifuge machine for 20 minutes at $1400 \mathrm{rpm}$. After this stage, "clot-activator gel" was separated from the blood serum. The gel kept chemical and biochemical components of the serum unchanged for a long time. Then, the serums were transferred to a micro-tube for measuring the TnI serum levels by Elisa method. The data obtained by ELISA reading (light absorption rate per well) were noted in the vertical axis of TnI standard curve. Then, they were extended until we reached the curve. The point was connected to the horizontal axis to achieve the TnI levels in $\mathrm{ng} / \mathrm{ml}$ or $\mu \mathrm{g} / \mathrm{l}$ unit (Figure 1).

\section{Histopathological evaluation}

The rats were anesthetized with ketamine and xylazine. After removal of the heart from the chest, the tissues were fixed with $10 \%$ neutral buffered formalin and then placed in paraffin. All parts of the heart were cut ( 5 


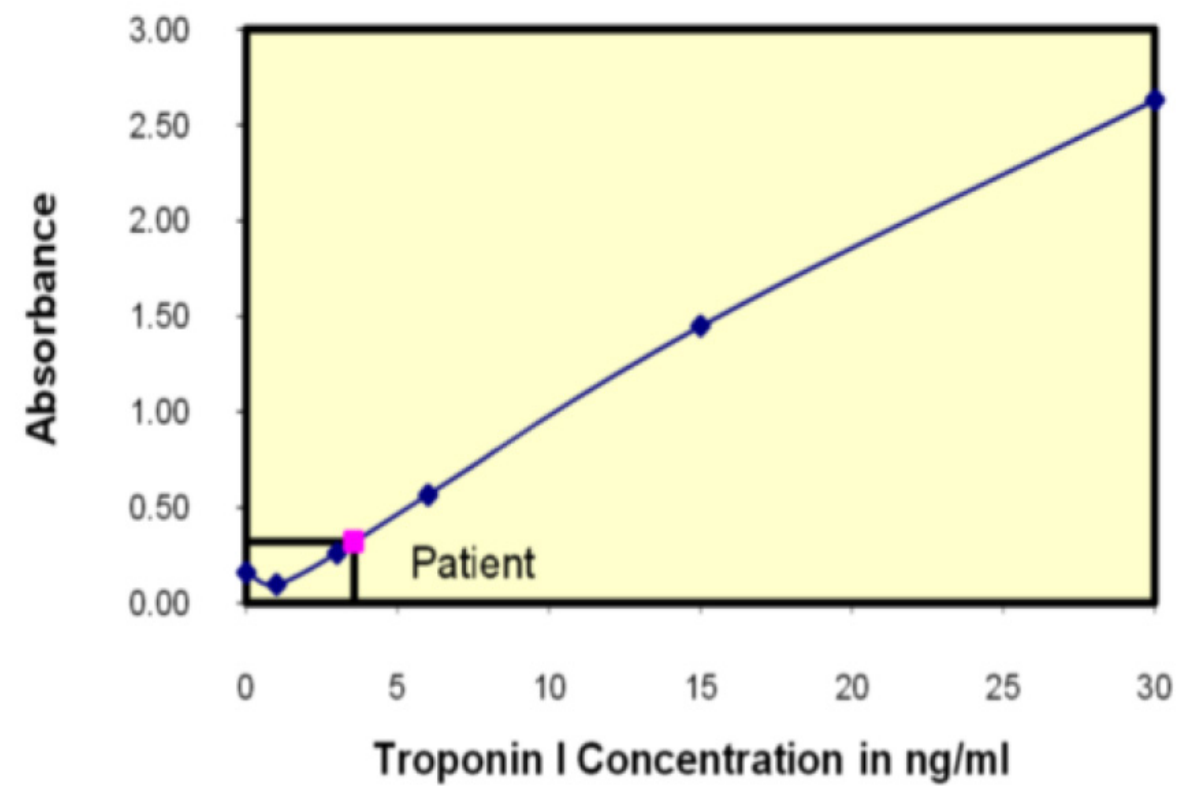

Figure 1: The standard curve of Troponin I

$\mu \mathrm{m})$, processed, and dyed with Hematoxylin and Eosin ( $\mathrm{H}$ and $\mathrm{E})$, Masson's trichrome (MTC). All histopathological studies were performed at the unit of pathology, Faghihi teaching hospital, Shiraz, Iran. The light microscope (Olympus BX41TF, Japan) was used for blinded histopathological evaluation, using a semi-quantitative scoring system for the extent and severity of histological parameters. According to the three layers of the heart, the tissues were examined and different items were evaluated to describe the heart injury induced by radiation, including inflammation, pericardial effusion, thrombus, macrophage, myocyte necrosis, fibrosis of myocardium, fibrosis of endocardium, myocardial degeneration, epicardial thickness, vascular leakage, and plaque. The degree of tissue damage was scaled from G0 to G2: G0= no injury, G1= moderate and $\mathrm{G} 2=$ severe.

\section{Statistical analysis}

A statistics software package (SPSS ${ }^{\circledR}$ for Windows version 19, Chicago, USA) was used for data analysis. Biochemical assay was ana- lyzed using ANOVA test and post hoc Tukey honest significant difference (HSD). Besides, histopathological evaluations were performed using the Pearson Chisquare test. Survival rate was evaluated using Kaplan-Meier method.

\section{Results}

The effect of HES on the weight of rats

First, Blood samples were collected from branch A (cardiac biomarker study) of rats after seven days; second, they were excluded from the review cycle and weight was taken from the branch $\mathrm{B}$ (chronic effects) twelve days after starting the test. Then, using analysis of covariance (ANOVA) and test Tukey post hoc, the average weights were compared in different groups. Significant differences in weight were found in groups of rats.

During the first twelve days after irradiation, a decrease in average weights was observed in the studied groups although it was not statistically significant $(p>0.05)$. In the following days, the mean weight increased in the study 
groups.

At the end, the average weights in groups of 20 Gy radiation and the group receiving HES/ radiation were compared to the same groups whose weights had declined on the $48^{\text {th }}$ day. However, there was no statistically significant difference $(\mathrm{p}>0.05)$.

During the final days, the weight of the radiation group was significantly less than that of the control group $(p=0.003)$. This factor in HES/radiation group was significantly more than that of radiation group $(\mathrm{p}=0.038)$.
At any stage of weight gain, there was no significant difference between the control and HES groups $(\mathrm{p}=0.002)$.

The effect of HES on the survival rate of rats

The results of the body weights and survival rate of the control and experimental groups, which were evaluated 8 weeks after local mediastinum irradiation, are shown in Figures 2 and 3.

The rats were administered into $100 \mathrm{mg} / \mathrm{kg} /$

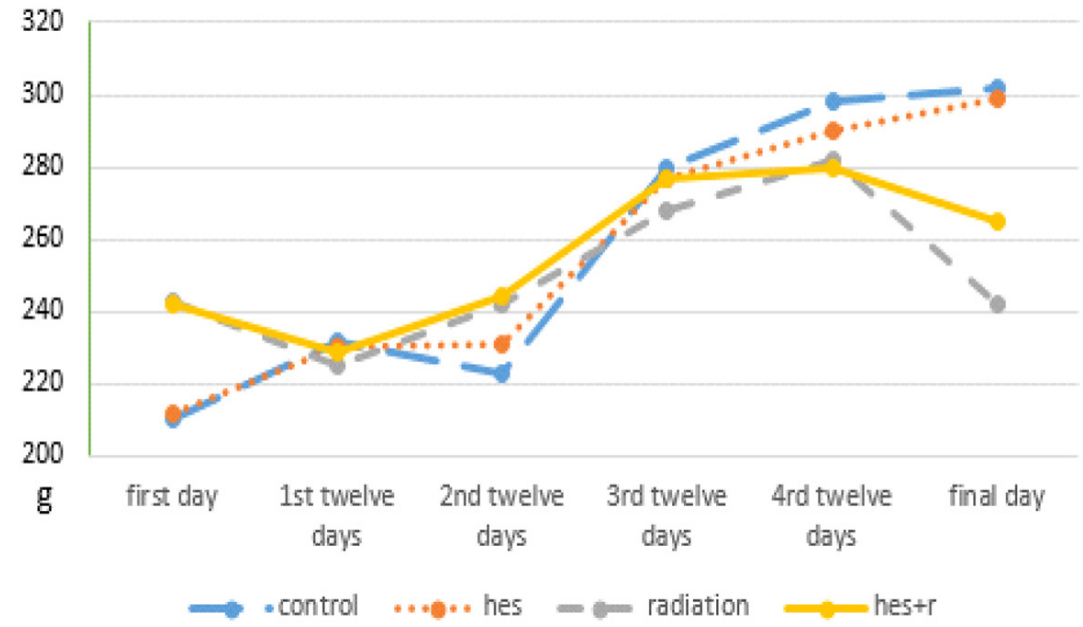

Figure 2: Weight of control and intervention groups

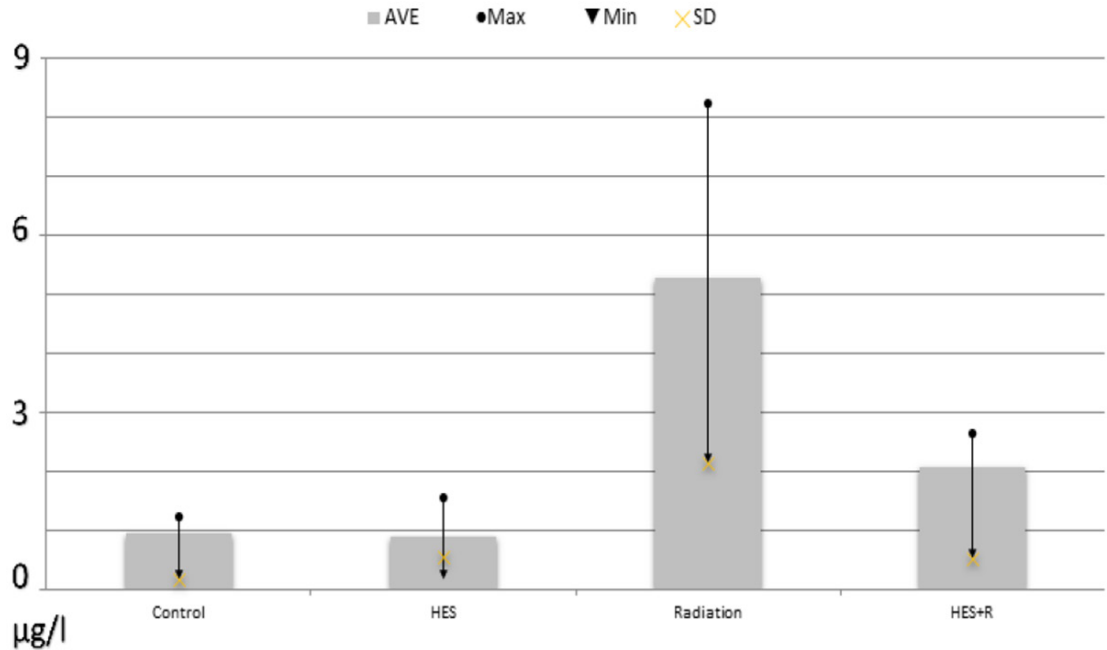

Figure 3: The survival rate of the control and intervention groups 
day of HES for 7 consecutive days before irradiation and monitored daily for 60 days. The results indicated that the rats, receiving $20 \mathrm{~Gy}$ radiation, had the symptoms of discomfort, which are known by the reduction in physical activity, water and food uptake. In addition, this group showed some signs, including weight loss, irritability, and epilation with a median survival time of 55 days. However, the rats that were pre-administered with HES, had less signs of radiation sickness and enhanced physical activity, body weight, and survival rate.

The effect of HES on the TnI serum level of rats

Sampling was performed from branch A of animals, including control group, HES group that received only $100 \mathrm{mg} / \mathrm{kg} / \mathrm{d}$ HES for 7 days continuously, Radiation group that was irradiated with one single dose of 20 Gy and HES $+\mathrm{R}$ group that was treated with HES and 4 to 6 hours after the last irradiation. TnI-derived samples were measured by ELISA. The measured values are shown in the Table 1:

The results showed that the average concentration of TnI in the radiation group significantly increased in comparison to control group ( $<<0.05)$ (Figure 4).
Histopathological damage of the heart tissue 24 hours after exposure

The histopathological examination of the heart sections of the control, HES, radiation, and $\mathrm{HES}+\mathrm{R}$ groups 24 hours post-irradiation was evaluated. The descriptive factors were examined, including the presence of inflammation, thrombus, myocyte necrosis, fibrosis of myocardium, fibrosis of endocardium, fibrosis of epicardium, vascular damage, Myocardial degeneration, epicardial thickness, pericardial effusion, decreased microvascular, vascular leakage, macrophage, and plaque.

Histopathological analysis of the rats' heart tissue after 24 hours in the intervention and control groups was performed using chi-square test. Despite moderate damage in a number of irradiated rats, no significant difference was

Table 1: Troponin average and standard deviation in the groups studied

\begin{tabular}{cccc} 
& Average \pm SD & Max & Min \\
\hline Control & $0.95 \pm 0.17$ & 1.22 & 0.63 \\
\hline HES & $0.88 \pm 0.56$ & 1.54 & 0.17 \\
\hline Radiation & $5.31 \pm 2.13$ & 8.24 & 2.81 \\
\hline HES+R & $2.07 \pm 0.53$ & 2.65 & 1.09
\end{tabular}

HES: Hesperidin, SD: Standard Deviation

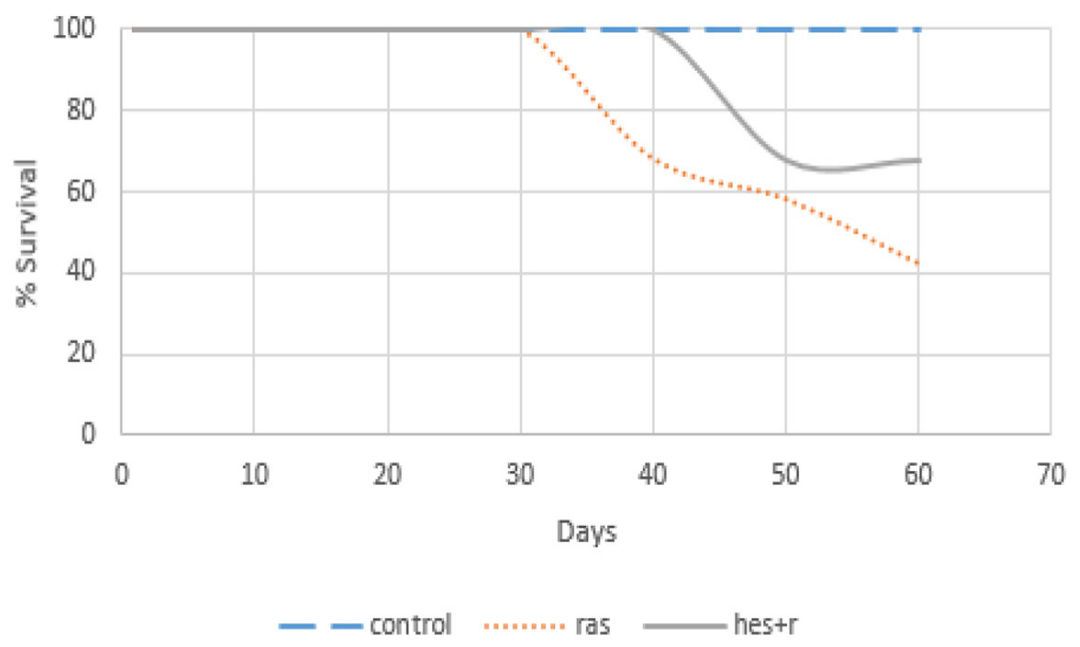

Figure 4: Serum levels of Troponin I (Tnl) in the control and intervention groups 
observed after statistical analysis $(p>0.05)$.

Histopathological damage of the heart tissue 8 weeks after exposure

The heart tissue of the second branch (B) of rats, including control, irradiated and treated

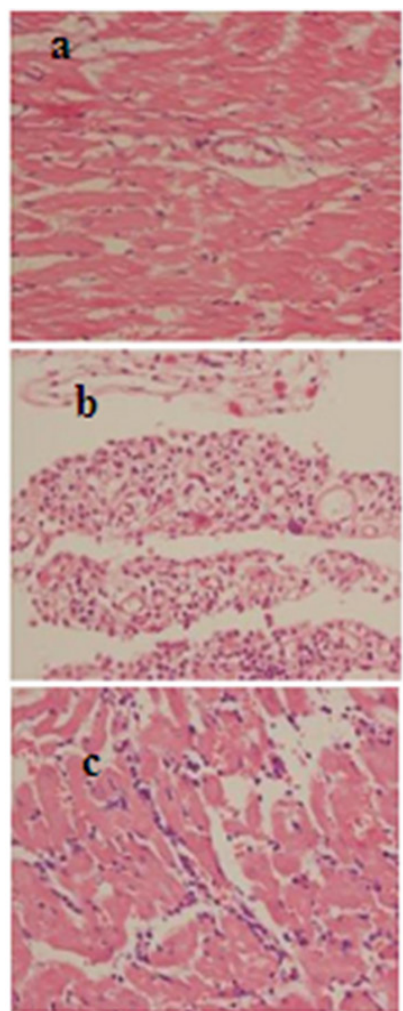

group with HES, 8 weeks after radiation, was extracted and analyzed by histopathology. To evaluate the histopathological changes, we used Fisher's exact test and Chi-square test. Histopathological results after 8 weeks were as follows (Figure 5):

1-Inflammation: According to the Table 2,
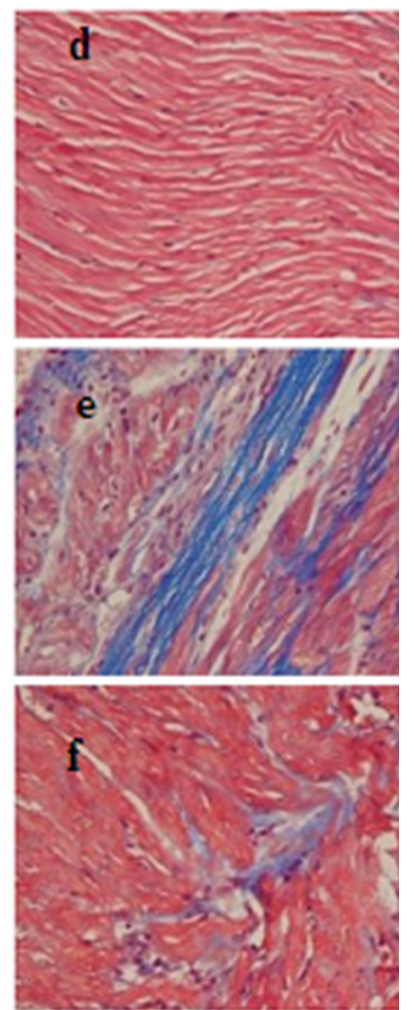

Figure 5: Histopathological findings of the rat hearts in the chronic phase- 8 weeks. H\&E (A to C), Trichrome masson (TCM) (D to F) stains of hearts were taken at $\times 400 . A$ : (H\&E) Control - Myocardial tissue and vascular bed are seen normal. B: (H\&E) Radiation group - Acute inflammation and accumulation of inflammatory cells are seen. C: (H\&E) Hesperidin (HES) + R group - Mild inflammation and the decreased infiltration of inflammatory cells are seen. D: (TCM) Control group - Myocardial tissue and vascular bed are seen normal. E: (TCM) Radiation group - Acute collagen deposition is seen. F: (TCM) HES+R group - Mild collagen deposition is seen.

Table 2: Inflammation 8 weeks after radiation in the groups studied

\begin{tabular}{cccc} 
& $\% \mathbf{~ 0 G}$ & $\% \mathbf{~} \mathbf{G}$ & $\mathbf{\%} \mathbf{~ G}$ \\
\hline Control & 100 & 0 & 0 \\
\hline Radiation & 20 & 50 & 30 \\
\hline HES+R & 100 & 0 & 0
\end{tabular}

HES: Hesperidin radiation successfully caused inflammation in irradiated rats' heart compared to the control group. This compression was statistically significant ( $p=0.008)$. Moreover, based on evaluation of the radiation effect and drug simultaneously, the group which received both drug and radiation in comparison with the group which received only radiation, had less inflammation 
and there was statistically significant difference between two groups ( $p=0.001)$. Then, by comparing the control and the HES $+\mathrm{R}$ groups, we found no significant different ( $p>0.016)$.

2-Thrombus: According to the result of Fisher's exact test, the effects of radiation in creating clots and the impact of the drug in reducing blood clots were studied. The results of this study did not show any significant difference between the investigated groups ( $\mathrm{p}>$ 0.016).

3-Myocyte necrosis: The effects of radiation on inducing necrosis and the drug in reducing the heart muscle cell death were evaluated. The results of this study did not show any significant differences between the studied groups ( $\mathrm{p}>0.016)$.

4-Fibrosis of myocardium: The effects of radiation on creating heart muscle fibrosis and the drug in reducing this damage were examined. The results of this study revealed no significant difference between the groups investigated $(p>0.016)$.

5-Fibrosis of endocardium: The effects of radiation on creating fibrosis of the endocardium and the drug in reducing fibrosis were examined. The results of this study revealed no significant difference between the investigated groups $(\mathrm{p}>0.016)$.

6-Myocardial degeneration: The effects of radiation on creating myocardial degeneration and the drug in reducing tissue degeneration were examined. The results of this study, also, showed no significant difference between the investigated groups ( $\mathrm{p}>0.016)$.

7-Epicardial thickness: The effects of radiation on the epicardial thickness and the drug

Table 3: Pericardial effusion 8 weeks after radiation in the groups studied

\begin{tabular}{cccc} 
& \% 0G & \% 1G & \% 2 $\mathbf{~}$ \\
\hline Control & 83.3 & 0 & 16.7 \\
\hline Radiation & 0 & 80 & 20 \\
\hline HES+R & 87.5 & 12.5 & 0
\end{tabular}

HES: Hesperidin in reducing this malformation were examined. The results of this study also showed no significant difference between the groups under the study ( $p>0.016)$.

8-Pericardial effusion: According to Table 3 , radiation increases the pericardial effusion and the difference between radiated and control groups was significant $(\mathrm{p}=0.001)$. Moreover, the evaluation of the effectiveness of drug and radiation simultaneously showed that the group which received radiation and drug, in comparison to the group which received only radiation, had less effusion and the difference between the two groups was statistically significant $(\mathrm{p}=0.001)$. Then, comparison of the control group and the group of rats that received both drug and radiation revealed that there was no significant difference between the groups in the amount of pericardial effusion $(p>0.016)$.

9-Vascular leakage: The effects of radiation on inducing vascular leakage and the drug in reducing leakage were examined. The results of this study did not show any significant difference between the studied groups ( $p>0.016)$.

10-Macrophage: The effects of radiation on increasing macrophage and the drug in reducing damage were examined. Based on results, it was proved that there is no significant difference between the investigated groups ( $\mathrm{p}>$ 0.016).

11-Plaque: According to Table 4, radiation increases the plaque and the difference between the radiation and control groups was significant $(p=0.001)$. Moreover, evaluating the effectiveness of drug and radiation simultaneously showed that the group which re-

Table 4: Vascular plaque 8 weeks after radiation in the groups studied

\begin{tabular}{cccc} 
& \% 0G & \% $\mathbf{~ G ~}$ & \% 2 $\mathbf{~}$ \\
\hline Control & 83.3 & 0 & 16.7 \\
\hline Radiation & 0 & 90 & 10 \\
\hline HES+R & 87.5 & 12.5 & 0
\end{tabular}

HES: Hesperidin 
ceived radiation and drug in comparison to the which received only radiation had less plaque and the difference between the two groups was statistically significant $(\mathrm{p}=0.001)$. Then, comparison of the control group and the group of rats that received both drug and radiation revealed that there was no significant difference between the groups for plaque $(\mathrm{p}>0.016)$.

\section{Discussion}

The major component of cells is water. Thus, when, the cells irradiated by ionizing radiation, radiolysis of water causes the production of primary radicals, which can react with oxygen or other molecules. This interaction results in the production of secondary radicals, including $\mathrm{H}_{2} \mathrm{O}_{2}$ and $\mathrm{O}_{2}^{-}$[15]. Free radicals are the main factor of radiation toxicity, hence an effective way to protect cells from damage induced by radiation is supplementation of antioxidants [16].

Radioprotectors have potential application in medical practices for protecting normal tissue during radiotherapy and reaching maximum therapy outcome. Furthermore, there are unplanned radiation sources such as nuclear accidents, background radiation or exposure during space missions that radioprotectors can play a major role in decreasing the consequences [17].

Different compounds have been tested as radioprotectors and Amifostine is a well-known radioprotector among them that the use of it is limited due to high cost, toxicity and side effects. Search for efficient radioprotector has been still needed. Recently, reports have shown that HES, a flavonoid, has many biological effects, including antimicrobial, antiinflammatory, antioxidant and anticarcinogenic effects which can significantly protect cells from DNA damage [17].

In this study, it was hypothesized that preadministration of HES for 7 consecutive days before exposure to a single dose of $20 \mathrm{~Gy}$ radiation, may ameliorate inflammatory responses and long-term pathological changes after exposure to radiation. The radioprotective effects of HES on myocardium after radiotherapy were observed in this study. The responsible mechanisms of this protection are not clear but there are evidence and reports that may explain these findings.

The irradiation of the rat's mediastinum with 20 Gy resulted in increased serum level of TnI and pathological changes in the heart tissue. Long-term changes are associated with the decreased survival rate, body weight, and increased heart tissue index. Besides, the irradiation of the heart tissue results in inflammation. The administration of HES decreases long-term damage. Pericardial effusion is seen after heart irradiation and the administration of HES ameliorated effusion.

Although in this study we found that exposure to 20 Gy using megavoltage irradiation photon beam can decrease the rat's mean weight down to $19.5 \%$ than non-irradiated rats. HES as a natural radioprotector plays its role perfectly; as it was observed, that drug improved the weight of rats in the HES $+\mathrm{R}$ group.

In addition, histopathological examination of cardiac vessels indicated that single fractional $\gamma$-irradiation could significantly increase the vascular plaque. There is not even any rat without plaque after 20Gy irradiation in the radiation group. Owing to oral administration of $100 \mathrm{mg} / \mathrm{kg} / \mathrm{d}$ HES as pre-figuration radioprotector per rat, only 12.5 percent of animals had vascular plaque. Accordingly, we can conclude that HES plays an important role in decreasing atherosclerosis created by radiation. Whereas in the new world a large number of deaths are caused by atherosclerosis, HES might successfully contribute to the community health.

Except for the above illness, other factors such as thrombus, myocyte necrosis and fibrosis of myocardium, fibrosis of endocardium, myocardial degeneration, epicardial thickness, vascular leakage and macrophage did not show any remarkable difference between the radiation and control groups. This might 
be caused by several reasons. Perhaps, radiation really does not have any effects on these factors, or because inflammation and plaque appear in the acute level but necrosis, fibrosis and degeneration develop in the chronic phases. This study terminated in 8 weeks after irradiation. It is suggested that in longer studies, more factors will show changes under the influence of radiation.

Since antioxidant effect and free radical scavenging of flavonoids is reported in many researches, there are several studies exploring radioprotection effect of HES at different conditions, including in vivo or in vitro, using different doses and observing various organs [17-23].

Pradeep et al. at 2008 reported, "The $\gamma$-irradiation induced toxic effects were dramatically and dose-dependently inhibited by HES treatment as observed by the restoration in the altered levels of the marker enzymes, lipid peroxidation, enzymatic and non-enzymatic antioxidants" and they refer this finding to its ability of membrane stabilization and free radical scavenging [15].

Hosseinimehr et al. investigated the protective effect of HES against genotoxicity induced by $\gamma$-irradiation in cultured blood lymphocytes from human. The data exhibited that "the maximum protection and decrease in frequency of micronuclei (33\%) was observed at $1 \mathrm{~h}$ after ingestion of HES". The author believes that "these data have important application for the protection of human lymphocytes from the genetic damage and side effects induced by $\gamma$-irradiation in patients undergoing radiotherapy" [24].

Kalpana et al. evaluated radioprotective efficacy of HES by performing various biochemical estimations, DNA fragmentation assay, comet assay and histopathological studies in the liver of mice. They observed that "pre-administration of HES restored the antioxidant status to near normal and decreased the levels of lipid peroxidative index, DNA damage and comet parameters" [16].
Moustafa ahmed et al. at their study showed that HES has a radioprotective effect against $\gamma$-irradiation-induced oxidative stress and biomechanical properties of bone in rats and suggested that "HES may serve as a potential protective agent against $\gamma$-irradiation-induced cardio-nephrotoxicity via enhancing the antioxidant activity" [25].

Although we suggest more clinical trials to evaluate the feasibility of the utilization of HES as a promising radio protector, further studies are useful to clarify the molecular mechanisms of HES protection effects.

\section{Conclusion}

In conclusion, this study showed that pretreatment with HES results in the reduction of myocardial damage and TnI level after mediastinal radiotherapy. These findings can help the patients with malignant tumor undergoing radiotherapy and HES is a promising candidate for radiation protection.

\section{Acknowledgment}

This paper has been extracted from the results of the MSc thesis of Somayeh Sajadi (No. 9074) that was funded by research council at Shiraz University of Medical Sciences. We would like to thank Mohammad Amin Mosleh Shirazi from the Department of Radiotherapy in Namazi hospital in Shiraz for his help with the irradiation of the animals and we really appreciate Masood Hossainzadeh from the Department of pathology for his help in histopathological study.

\section{Conflict of Interest}

None

\section{References}

1. Fuster V, Voute J. MDGs: chronic diseases are not on the agenda. Lancet. 2005;366:1512-4. doi: 10.1016/S0140-6736(05)67610-6. PubMed PMID: 16257330.

2. Topol EJ, Leya F, Pinkerton CA, Whitlow PL, Hofling B, Simonton CA, et al. A comparison of directional atherectomy with coronary angio- 
Hespiridin Modulates Heart Tissue Injury of Irradiated Rats

plasty in patients with coronary artery disease. N Engl J Med. 1993;329:221-7. doi: 10.1056/ NEJM199307223290401. PubMed PMID: 8316266.

3. Dale W, Gray LH, Meredith W. The inactivation of an enzyme (Carboxypeptidase) by $\mathrm{X}$-and $\alpha$-radiation. Philosophical Transactions of the Royal Society of London Series A. Mathematical and Physical Sciences. 1949;242:33-62. doi: 10.1098/rsta.1949.0004.

4. Hosseinimehr SJ, Tavakoli H, Pourheidari G, Sobhani A, Shafiee A. Radioprotective effects of citrus extract against gamma-irradiation in mouse bone marrow cells. J Radiat Res. 2003;44:237-41. PubMed PMID: 14646227.

5. Tiwari AK. Imbalance in antioxidant defence and human diseases: Multiple approach of natural antioxidants therapy. Curr Sci. 2001:1179-87.

6. Lee Y-S, Huh J-Y, Nam S-H, Moon S-K, Lee S-B. Enzymatic bioconversion of citrus hesperidin by Aspergillus sojae naringinase: Enhanced solubility of hesperetin-7-0-glucoside with in vitro inhibition of human intestinal maltase, HMG-CoA reductase, and growth of Helicobacter pylori. Food Chem. 2012;135:2253-9. doi: 10.1016/j.foodchem.2012.07.007.

7. Babuin L, Jaffe AS. Troponin: the biomarker of choice for the detection of cardiac injury. CMAJ. 2005;173:1191-202.

8. Horwich TB, Patel J, MacLellan WR, Fonarow GC. Cardiac troponin I is associated with impaired hemodynamics, progressive left ventricular dysfunction, and increased mortality rates in advanced heart failure. Circulation. 2003;108:833-8. doi: 10.1161/01.CIR.0000084543.79097.34. PubMed PMID: 12912820.

9. Tommasini S, Calabro ML, Stancanelli R, Donato $P$, Costa C, Catania S, et al. The inclusion complexes of hesperetin and its 7-rhamnoglucoside with (2-hydroxypropyl)-beta-cyclodextrin. J Pharm Biomed Anal. 2005;39:572-80. doi: 10.1016/j. jpba.2005.05.009. PubMed PMID: 15985355.

10. Emim JA, Oliveira AB, Lapa AJ. Pharmacological evaluation of the anti-inflammatory activity of a citrus bioflavonoid, hesperidin, and the isoflavonoids, duartin and claussequinone, in rats and mice. J Pharm Pharmacol. 1994;46:118-22. doi: 10.1111/j.2042-7158.1994.tb03753.x. PubMed PMID: 8021799.

11. Garg A, Garg S, Zaneveld LJ, Singla AK. Chemistry and pharmacology of the Citrus bioflavonoid hesperidin. Phytother Res. 2001;15:655-69. doi: 10.1002/ptr.1074. PubMed PMID: 11746857.

12. Bayes-Genis A, Barallat J, Pascual D, Nunez J, Minana G, Sanchez-Mas J, et al. Rapid Fire 1Acute Heart Failure. Eur J Heart Fail. 2015;17:5441.

13. Serin M, Gulbas H, Gurses I, Erkal HS, Yucel N. The histopathological evaluation of the effectiveness of melatonin as a protectant against acute lung injury induced by radiation therapy in a rat model. Int J Radiat Biol. 2007;83:187-93. doi: 10.1080/09553000601129093. PubMed PMID: 17378527.

14. Hosseinimehr S, Nemati A. Radioprotective effects of hesperidin against gamma irradiation in mouse bone marrow cells. The British Journal of Radiology. 2006;79:415-8.

15. Pradeep K, Park SH, Ko KC. Hesperidin a flavanoglycone protects against gamma-irradiation induced hepatocellular damage and oxidative stress in Sprague-Dawley rats. Eur $J$ Pharmacol. 2008;587:273-80. doi: 10.1016/j. ejphar.2008.03.052. PubMed PMID: 18485345.

16. Kalpana KB, Devipriya N, Srinivasan M, Vishwanathan $P$, Thayalan K, Menon VP. Evaluating the radioprotective effect of hesperidin in the liver of Swiss albino mice. Eur J Pharmacol. 2011;658:20612. doi: 10.1016/j.ejphar.2011.02.031. PubMed PMID: 21371459.

17. Kuntic V, Brboric J, Holclajtner-Antunovic I, Uskokovic-Markovic S. Evaluating the bioactive effects of flavonoid hesperidin--a new literature data survey. Vojnosanit Pregl. 2014;71:60-5. doi: 10.2298/vsp1401060k. PubMed PMID: 24516992.

18. Kalpana K, Devipriya N, Srinivasan M, Menon VP. Investigation of the radioprotective efficacy of hesperidin against gamma-radiation induced cellular damage in cultured human peripheral blood lymphocytes. Mutation Research/Genetic Toxicology and Environmental Mutagenesis. 2009;676:54-61. doi: 10.1016/j.mrgentox.2009.03.005.

19. Dominello MM, Fountain MD, Rothstein SE, Cannon AC, Abernathy LM, Hoogstra D, et al. Radiation injury to cardiac arteries and myocardium is reduced by soy isoflavones. Journal of Radiation Oncology. 2017;6:307-15. doi: 10.1007/s13566017-0301-z.

20. Park SH, Pradeep K, Ko KC. Protective effect of hesperidin against $\gamma$-radiation induced oxidative stress in Sprague-Dawley rats. Pharm Biol. 2009;47:9407. doi: $10.1080 / 13880200902950793$.

21. Hozayen WG, Abou Seif $H$. Protective effects of rutin and hesperidin against doxorubicin-induced 
lipodystrophy and cardiotoxicity in albino rats. $J$ Am Sci. 2011;7:765-75.

22. Rezaeyan A, Fardid R, Haddadi GH, Takhshid MA, Hosseinzadeh M, Najafi M, et al. Evaluating Radioprotective Effect of Hesperidin on Acute Radiation Damage in the Lung Tissue of Rats. $J$ Biomed Phys Eng. 2016;6:165-74. PubMed PMID: 27853724. PubMed PMCID: PMC5106549.

23. Ghorbani Zh, Fardid R, Haddadi GH, Derakhshanfar A, Kouhpayeh A, Haddadi Z. Histopathologic Evaluation of Radio- Protective Effect of Hesperidin on the Liver of Sprague Dawely Rats. J Biomed Phys Eng. 2020;10(1):7-14. doi: 10.31661/jbpe. v0i0.832. PubMed PMID: 32158707. PubMed PMCID: PMC7036407.

24. Hosseinimehr SJ, Mahmoudzadeh A, Ahmadi A, Mohamadifar S, Akhlaghpoor S. Radioprotective effects of hesperidin against genotoxicity induced by gamma-irradiation in human lymphocytes. $\mathrm{Mu}$ tagenesis. 2009;24:233-5. doi: 10.1093/mutage/ gep001. PubMed PMID: 19193695.

25. Ahmed HM, Hussein MA, Alazonee AS. Radioprotective effect of hesperidin against gammairradiation-induced oxidative stress and biomechanical properties of bone in rats. Life Sci J. 2013;10:2857-65. 